\title{
EXCITATION OF AN ELASTIC HALF-SPACE BY A TIME-DEPENDENT DIPOLE-I. THE SURFACE DISPLACEMENTS DUE TO A SURFACE DIPOLE
}

\author{
O. F. AFAnDI and R. A. Scott \\ Engineering Mechanics Department, University of Michigan, Michigan
}

\begin{abstract}
The surface displacements due to a surface, time-dependent dipole in an elastic half-space are treated. Closed-form expressions are given for an arbitrarily oriented dipole with a ramp time-dependence. These expressions are analyzed numerically for a special dipole orientation for several values of the ramp rise-time.
\end{abstract}

\section{INTRODUCTION}

TRANSIENT elastic wave propagation in a homogeneous elastic half-space is treated, the waves being generated by a time-dependent dipole. Such a study is a contribution to nonaxisymmetric elastodynamics, an area of emerging interest (see Scott and Miklowitz [1]). It is also important in view of the fact that dipole sources can be regarded as simple earthquake models.

A substantial amount of work on elastic transients in a half-space has already been done. First motion studies involving various source models have been given by Knopoff and Gilbert $[2,3]$ and Burridge et al. $[4]$. Pekeris $[5,6]$ gave closed-form solutions for the surface displacements due to a vertical, surface point source with a Heaviside step timedependence. This same problem was treated by Chao et al. [7], who retained only the Rayleigh wave contribution in their numerical work. Lang [8], in work on a surface point load, gave numerical results for distinct surface events. Pekeris [9] also gave results in terms of integrals for the surface displacements due to a buried, vertical force with a Heaviside step time-dependence. In a later paper [10], he and Lifson evaluated the integrals numerically and gave results for several epicentral distances. Pinney [11] obtained the surface displacements due to impulsive, internal sources and torques. His results were in terms of integrals, information being obtained by numerical integration. The buried torque pulse was also treated by Pekeris and Longman [12]. They obtained the surface vertical displacements and evaluated the integrals that arose numerically. Chao [13] presented closed-form, surface results for the case of the surface of the half-space being loaded by a tangential point force with a Heaviside step time-dependence. A significant contribution was given by Aggarwal and Ablow $[14,15]$, who developed a method whereby surface results for arbitrary, buried sources can be obtained in terms of integrals. The works of Eason [16] and Mitra [17] should also be noted. Eason, using integral representations and a Cagniard-de Hoop method, obtained surface results and large-time results for the case of a suddenly applied, vertical, surface force. Mitra presented surface information on 
the displacements produced by time-dependent body forces. However, his solutions are in terms of integrals and no numerical information was given.

Little work has been done on the transients generated by dipole (and other multipole) sources. Chandra [18], using saddle-point methods, gave results on the surface phases generated by a double couple source with a Heaviside step time-dependence. The present paper treats the surface displacements produced by a surface dipole with a ramp timedependence. A major contribution is that closed-form expressions are given for the horizontal and vertical displacements for the case of an arbitrarily oriented dipole. The formal solutions are obtained using Fourier-Bessel superposition methods and the Laplace transformation. Inversion is achieved with the aid of contour integration techniques.

Numerical studies for several values of the ramp rise-time, are presented for a special dipole geometry.

\section{DEVELOPMENT OF THE SOLUTIONS}

Only shear faults are treated, that is (see Fig. 1)

$$
f_{1} n_{1}+f_{2} n_{2}+f_{3} n_{3}=0
$$

where the $n$ 's are the Cartesian components of a unit vector perpendicular to the fault plane and the $f$ 's are the Cartesian components of a unit force in the fault plane. A point dipole is an idealized model of such a fault. Cylindrical coordinates $r, \phi$ and $z$ are used with the origin at the dipole, which is a depth $d$ below the surface of the half-space and the $z$ axis pointed vertically downwards. In view of the very considerable amount of algebra involved, only an outline of the procedure will be given.

Following the development given by Haskell [19], it can be shown that the Laplacetransformed displacements $\bar{u}_{r}, \bar{u}_{\phi}$ and $\bar{u}_{z}$ due to a point dipole in an infinite medium, are, for $z<0, \dagger$

$$
\frac{4 \pi \rho p^{2}}{\bar{h}(p)} \bar{u}_{r}=-\frac{G_{1}}{4} B_{2}+B_{1}^{c} \cos \phi+B_{1}^{s} \sin \phi+B_{0}
$$

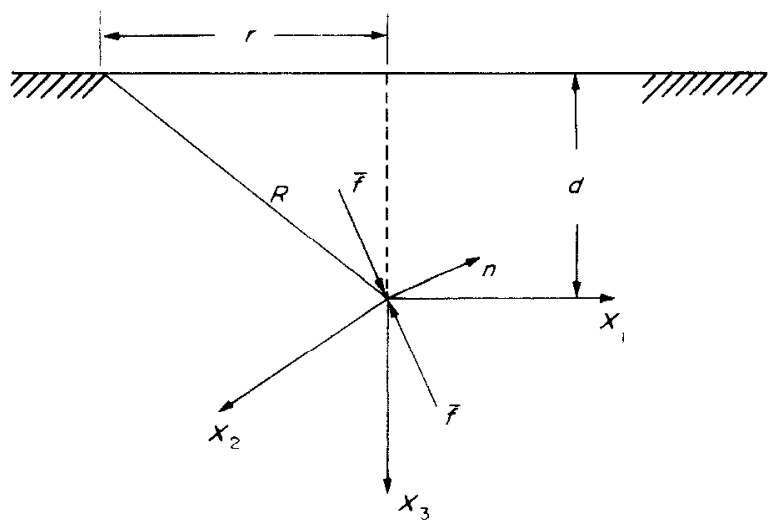

FiG. 1. Dipole geometry.

+ Since interest is ultimately in the surface displacements, only $z<0$ is considered in the sequel. 


$$
\begin{aligned}
& \frac{4 \pi \rho p^{2}}{\bar{h}(p)} \bar{u}_{\phi}=-\frac{G_{2}}{4} D_{2}+D_{1}^{c} \cos \phi+D_{1}^{s} \sin \phi+D_{0} \\
& \frac{4 \pi \rho p^{2}}{\bar{h}(p)} \bar{u}_{z}=\frac{G_{1}}{4} E_{2}+E_{1}^{c} \cos \phi+E_{1}^{s} \sin \phi-E_{0} \\
& G_{1}=2\left[\left(f_{2} n_{2}-f_{1} n_{1}\right) \cos 2 \phi-\left(f_{1} n_{2}+f_{2} n_{1}\right) \sin 2 \phi\right] \\
& G_{2}=-2\left[\left(f_{1} n_{2}+f_{2} n_{1}\right) \cos 2 \phi+\left(f_{2} n_{2}-f_{1} n_{1}\right) \sin 2 \phi\right] \\
& B_{2}=\int_{0}^{\infty} k^{2}\left(\frac{k^{2}}{\eta_{1}} \mathrm{e}^{z \eta_{1}}-\eta_{2} \mathrm{e}^{2 \eta_{2}}\right) J_{1}(k r) \mathrm{d} k-\frac{2}{r} \int_{0}^{\infty} k^{3}\left(\frac{\mathrm{e}^{z \eta_{1}}}{\eta_{1}}-\frac{\mathrm{e}^{z \eta_{2}}}{\eta_{2}}\right) J_{2}(k r) \mathrm{d} k \\
& B_{1}^{c}=-\left(f_{3} n_{1}+f_{1} n_{3}\right)\left[\int_{0}^{\infty} k^{3}\left(\mathrm{e}^{z \eta_{1}}-\mathrm{e}^{z \eta_{2}}\right) J_{0}(k r) \mathrm{d} k-\frac{1}{r} \int_{0}^{\infty} k^{2}\left(\mathrm{e}^{z \eta_{1}}-\mathrm{e}^{z \eta_{2}}\right) J_{1}(k r) \mathrm{d} k\right] \\
& +f_{1} n_{3} \frac{p^{2}}{c_{s}^{2}} \int_{0}^{\infty} k \mathrm{e}^{2 \eta_{2}} J_{0}(k r) \mathrm{d} k \\
& B_{1}^{\mathrm{s}}=-\left(f_{2} n_{3}+f_{3} n_{2}\right)\left[\int_{0}^{\infty} k^{3}\left(\mathrm{e}^{z \eta_{1}}-\mathrm{e}^{z \eta_{2}}\right) J_{0}(k r) \mathrm{d} k-\frac{1}{r} \int_{0}^{\infty} k^{2}\left(\mathrm{e}^{z \eta_{1}}-\mathrm{e}^{z \eta_{2}}\right) J_{1}(k r) \mathrm{d} k\right] \\
& +f_{2} n_{3} \frac{p^{2}}{c_{s}^{2}} \int_{0}^{\infty} k \mathrm{e}^{2 \eta_{2}} J_{0}(k r) \mathrm{d} k \\
& B_{0}=\frac{f_{3} n_{2}}{2} \int_{0}^{\infty} k^{2}\left[3 \eta_{2} \mathrm{e}^{z \eta_{2}}-\left(3 \eta_{1}-\frac{p^{2}}{\eta_{1} c_{d}^{2}}\right) \mathrm{e}^{z \eta_{1}}\right] J_{1}(k r) \mathrm{d} k \\
& D_{2}=\frac{2}{r} \int_{0}^{\infty} k^{2}\left(\frac{\mathrm{e}^{z \eta_{1}}}{\eta_{1}}-\frac{\mathrm{e}^{z \eta_{2}}}{\eta_{2}}\right) J_{2}(k r) \mathrm{d} k-\frac{p^{2}}{c_{d}^{2}} \int_{0}^{\infty} \frac{k^{2}}{\eta_{2}} \mathrm{e}^{z \eta_{2}} J_{1}(k r) \mathrm{d} k \\
& D_{1}^{c}=-\left(\frac{f_{3} n_{2}+f_{2} n_{3}}{r}\right) \int_{0}^{\infty} k^{2}\left(\mathrm{e}^{z \eta_{1}}-e^{z \eta_{2}}\right) J_{1}(k r) \mathrm{d} k+f_{2} n_{3} \frac{p^{2}}{c_{s}^{2}} \int_{0}^{\infty} k \mathrm{e}^{z \eta_{2}} J_{0}(k r) \mathrm{d} k \\
& D_{1}^{s}=\left(\frac{f_{3} n_{1}+f_{1} n_{3}}{r}\right) \int_{0}^{\infty} k^{2}\left(\mathrm{e}^{z \eta_{1}}-e^{z \eta_{2}}\right) J_{1}(k r) \mathrm{d} k-f_{1} n_{3} \frac{p^{2}}{c_{s}^{2}} \int_{0}^{\infty} k \mathrm{e}^{z \eta_{2}} J_{0}(k r) \mathrm{d} k \\
& D_{0}=\frac{1}{2}\left(f_{1} n_{2}-f_{2} n_{1}\right) \int_{0}^{\infty} \frac{k^{2}}{\eta_{2}} \mathrm{e}^{z \eta_{2}} J_{1}(k r) \mathrm{d} k \\
& E_{2}-\int_{0}^{\infty} k^{3}\left(\mathrm{e}^{z \eta_{2}}-\mathrm{e}^{z \eta_{1}}\right) J_{2}(k r) \mathrm{d} k \\
& E_{1}^{c}=\left(f_{1} n_{3}+f_{3} n_{1}\right) \int_{0}^{\infty} k^{2}\left(\eta_{2} \mathrm{e}^{z \eta_{2}}-\eta_{1} \mathrm{e}^{z \eta_{1}}\right) J_{1}(k r) \mathrm{d} k-f_{3} n_{1} \frac{p^{2}}{c_{s}^{2}} \int_{0}^{\infty} \frac{k^{2}}{\eta_{2}} \mathrm{e}^{z \eta_{2}} J_{1}(k r) \mathrm{d} k \\
& E_{1}^{s}=\left(f_{2} n_{3}+f_{3} n_{2}\right) \int_{0}^{\infty} k^{2}\left(\eta_{2} \mathrm{e}^{z \eta_{2}}-\eta_{1} \mathrm{e}^{z \eta_{1}}\right) J_{1}(k r) \mathrm{d} k \\
& -f_{3} n_{2} \frac{p^{2}}{c_{s}^{2}} \int_{0}^{\infty} \frac{k^{2}}{\eta_{2}} \mathrm{e}^{z \eta_{2}} J_{1}(k r) \mathrm{d} k
\end{aligned}
$$




$$
\begin{aligned}
E_{0}=\frac{f_{3} n_{3}}{2} \int_{0}^{\infty} k\left[3 k^{2} \mathrm{e}^{2 \eta_{2}}-\left(2 \frac{p^{2}}{c_{d}^{2}}-3 k^{2}\right) \mathrm{e}^{2 \eta_{1}}\right] J_{0}(k r) \mathrm{d} k \\
\eta_{1}=\sqrt{\left(k^{2}+\frac{p^{2}}{c_{d}^{2}}\right),} \quad \eta_{2}=\sqrt{ }\left(k^{2}+\frac{p^{2}}{c_{s}^{2}}\right) \\
c_{d}^{2}=(\lambda+2 \mu) / \rho, \quad c_{s}^{2}=\mu / \rho .
\end{aligned}
$$

$\lambda$ and $\mu$ being the Lamé constants, $\rho$ the density, $h(t)$ the source time-dependence, the $J$ 's Bessel functions of the first kind and the bar denotes the Laplace transform, parameter $p$. In arriving at (1)-(3), the integral representations

$$
\frac{1}{\sqrt{\left(z^{2}+r^{2}\right)}} \exp \left[-\frac{p \sqrt{ }\left(z^{2}+r^{2}\right)}{c_{d}\left(c_{s}\right)}\right]=\int_{0}^{\infty} \frac{k}{\eta_{1}} \exp \left[-|z| \eta_{1}\left(\eta_{2}\right)\right] J_{0}(k r) \mathrm{d} k
$$

were used. As (4) shows, branch points arise on the imaginary p-axis. The branches that were chosen were such that $\operatorname{Re} \eta_{1}>0, \operatorname{Re} \eta_{2}>0$, when $\operatorname{Re} p>0, \operatorname{Re}$ denoting real part.

The solutions to the half-space problem are obtained by using (1)-(3) to suitably adjust general solutions to the equations of motion, the resulting expressions being in turn adjusted so that the surface of the half-space is stress-free. Following Scott and Miklowitz [1] it can be shown that quite general solutions to the transformed equation of motion are

$$
\begin{aligned}
-\frac{4 \pi \rho p^{2}}{\bar{h}(p)} \bar{u}_{r}^{n}= & \cos n \phi \int_{0}^{\infty}\left[k \chi_{1}^{n c} J_{n-1}(k r)-\frac{n}{r}\left(\chi_{1}^{n c}+\chi_{3}^{n c}\right) J_{n}(k r)\right] \mathrm{d} k \\
& +\sin n \phi \int_{0}^{\infty}\left[k \chi_{1}^{n s} J_{n-1}(k r)-\frac{n}{r}\left(\chi_{1}^{n s}+\chi_{3}^{n s}\right)\right] J_{n}(k r) \mathrm{d} k \\
-\frac{4 \pi \rho p^{2}}{\bar{h}(p)} \bar{u}_{\phi}^{n}= & \sin n \phi \int_{0}^{\infty}\left[k \chi_{3}^{n c} J_{n-1}(k r)-\frac{n}{r}\left(\chi_{1}^{n c}+\chi_{3}^{n c}\right) J_{n}(k r)\right] \mathrm{d} k \\
& -\cos n \phi \int_{0}^{\infty}\left[k \chi_{3}^{n s} J_{n-1}(k r)-\frac{n}{r}\left(\chi_{1}^{n s}+\chi_{3}^{n s}\right) J_{n}(k r)\right] \mathrm{d} k \\
-\frac{4 \pi \rho p^{2}}{\bar{h}(p)} \bar{u}_{z}^{n}= & \cos n \phi \int_{0}^{\infty} \chi_{2}^{n c} J_{n}(k r) \mathrm{d} k+\sin n \phi \int_{0}^{\infty} \chi_{2}^{n s} J_{n}(k r) \mathrm{d} k
\end{aligned}
$$

where $n$ is an integer, provided

$$
\begin{gathered}
\chi_{1}^{n c(s)}=\frac{\mathrm{d} \Phi_{2}^{n c(s)}}{\mathrm{d} z}-\Phi_{1}^{n c(s)} \\
\chi_{2}^{n c(s)}=-\frac{\mathrm{d} \Phi_{1}^{n c(s)}}{\mathrm{d} z}+k^{2} \Phi_{2}^{n c(s)} \\
\frac{\mathrm{d}^{2} \Phi_{j}^{n c(s)}}{\mathrm{d} z^{2}}-\eta_{j}^{2} \Phi_{j}^{n c(s)}=0, \quad j=1,2, \\
\frac{\mathrm{d}^{2} \chi_{3}^{n c(s)}}{\mathrm{d} z^{2}}-\eta_{2}^{2} \chi_{3}^{n c(s)}=0 .
\end{gathered}
$$

Solutions to (8)-(11) are readily obtained. On substituting these solutions, which involve arbitrary constants, into $(5)-(7)$, a general family of solutions to the transformed 
displacement equations of motion is obtained. $\dagger$ Not all members of this family are required for the problem at hand. In fact, since no $\phi$-boundary arises, the $\phi$-dependence in the solutions must be the same as that of the source. Hence only the $n=0,1$ and 2 terms in (5)(11) are retained. The arbitrary constants involved are evaluated using the following procedure : (i) Terms corresponding to (1)-(3) are matched. (ii) Use is made of the transformed stress-displacement relations and the fact that the half-space boundary is stressfree. On noting the linear independence of the trigonometric functions, several conditions arise. For example

$$
\bar{\sigma}_{z r}=0, \quad \bar{\sigma}_{z \theta}=0, \quad z=-d
$$

lead to, in part

$$
\begin{aligned}
& \int_{0}^{\infty}\left\{\frac{\mathrm{d} J_{2}(k r)}{\mathrm{d} r}\left[f_{2}(k, z)+\frac{\mathrm{d} f_{1}(k, z)}{\mathrm{d} z}\right]_{z=-d}-\frac{2}{r} J_{2}(k r)\left[\frac{\mathrm{d} f_{3}(k, z)}{\mathrm{d} z}\right]_{z=-d}\right\} \mathrm{d} k=0 \\
& \int_{0}^{\infty}\left\{\frac{\mathrm{d} J_{2}(k r)}{\mathrm{d} r}\left[\frac{\mathrm{d} f_{3}(k, z)}{\mathrm{d} z}\right]_{z=-d}+\frac{2}{r} J_{2}(k r)\left[f_{2}(k, z)+\frac{\mathrm{d} f_{1}(k, z)}{\mathrm{d} z}\right]_{z=-d}\right\} \mathrm{d} k=0
\end{aligned}
$$

where the functions $f_{1}, f_{2}$ and $f_{3}$ involve arbitrary constants. Multiplying (12) by $r / 2$, differentiating the result with respect to $r$ and adding to (13), can be shown to give

$$
\left(f_{2}+\frac{\mathrm{d} f_{1}}{\mathrm{~d} z}\right)_{z=-d}=0, \quad\left(\frac{\mathrm{d} f_{3}}{\mathrm{~d} z}\right)_{z=-d}=0 .
$$

It should be noted here that in [1] it is stated that equations such as (14) follow immediately from equations such as (12). However, in view of the recursion relation

$$
\frac{2}{r} J_{2}(k r)+\frac{\mathrm{d}}{\mathrm{d} r} J_{2}(k r)=k J_{1}(k r)
$$

the right side of which has an infinite number of zeros in the range of integration, the procedure of setting the integrands in (12) and (13) to zero separately as a general one is suspect. But, as occurs in [1], the method can lead to correct results in certain circumstances, such as restricting the manner in which the boundary shear stresses can vary. The last author in particular is grateful to Dr. K. Viswanathan who pointed out the current, more general method in a private communication.

Proceeding in the above fashion, enough conditions for the determination of the arbitrary constants can be found. Once the constants have been obtained, the transformed field quantities can be found. After considerable algebra, the transformed radial displacement $\ddagger$ can be shown to be

$$
\begin{gathered}
\bar{u}_{r}=\bar{u}_{r}^{0}+\bar{u}_{r}^{1}+\bar{u}_{r}^{2} \\
\frac{4 \pi \rho p^{2}}{h(p)} \bar{u}_{r}^{0}=-\frac{n_{3} f_{3}}{2} \int_{0}^{\infty} k^{2}\left(L_{1}+L_{2}\right) J_{1}(k r) \mathrm{d} k
\end{gathered}
$$

† The transformed stresses can be found on using the transformed stress-displacement relations.

$\ddagger$ For the sake of brevity, the subsequent procedure is illustrated only for $\bar{u}_{r}$. 


$$
\begin{aligned}
\frac{4 \pi \rho p^{2}}{\bar{h}(p)} \bar{u}_{r}^{1}= & 2\left(f_{1} n_{3}+f_{3} n_{1}\right) \cos \phi\left[\int_{0}^{\infty}\left(L_{3}+L_{4}+L_{5}\right) \frac{k J_{2}(k r)}{D} \mathrm{~d} k\right. \\
& \left.-\int_{0}^{\infty}\left(L_{4}+L_{6}+L_{7}\right) \frac{k J_{0}(k r)}{D} \mathrm{~d} k\right] \\
& +2\left(f_{2} n_{3}+f_{3} n_{2}\right) \sin \phi\left[\int_{0}^{\infty}\left(L_{5}+L_{8}+L_{9}\right) \frac{k J_{2}(k r)}{D} \mathrm{~d} k\right. \\
& \left.-\int_{0}^{\infty}\left(L_{9}+L_{10}+L_{11}\right) \frac{k J_{0}(k r)}{D} \mathrm{~d} k\right]
\end{aligned}
$$

$$
\frac{4 \pi \rho p^{2}}{\bar{h}(p)} \bar{u}_{r}^{2}=\frac{G_{1}}{2}\left[\int_{0}^{\infty}\left(L_{12}+L_{13}+L_{14}\right) \frac{k^{2} J_{1}(k r)}{\eta_{1} D} \mathrm{~d} k-\int_{0}^{\infty}\left(L_{13}+L_{15}+L_{16}\right) \frac{k^{2} J_{3}(k r)}{\eta_{1} D} \mathrm{~d} k\right]
$$

$$
L_{1}=-\frac{3 \eta_{2}}{D}\left[g \mathrm{e}^{-(2 d+z) \eta_{2}}+\mathrm{g} \frac{2 \eta_{1}^{2}+k^{2}}{3 \eta_{1} \eta_{2}} \mathrm{e}^{-(2 d+z) \eta_{1}}-\frac{4}{3}\left(k^{2}+\eta_{2}^{2}\right)\left(k^{2}+2 \eta_{1}^{2}\right) \mathrm{e}^{-d \eta_{1}-(d+z) \eta_{2}}\right]
$$$$
L_{2}=\frac{12 k \eta_{2}^{2}}{D}\left(k^{2}+\eta_{2}^{2}\right) \mathrm{e}^{-\mathrm{d} \eta_{2}-(d+z) \eta_{1}}-3 \eta_{2} \mathrm{e}^{z \eta_{2}}+\frac{2 \eta_{1}^{2}+k^{2}}{\eta_{1}} \mathrm{e}^{\eta_{1} z}
$$$$
L_{3}=\left[A_{1} \frac{p^{2}}{c_{s}^{2}} D-g\left(k^{2}+A_{1} \frac{p^{2}}{c_{\mathrm{s}}^{2}}\right)\right] \mathrm{e}^{-(2 d+z) \eta_{2}}-k^{2} g \mathrm{e}^{-(2 d+z) \eta_{1}}
$$$$
L_{4}=4 k^{2} \eta_{1} \eta_{2}\left(k^{2}+\eta_{2}^{2}\right) \mathrm{e}^{-\mathrm{d} \eta_{1}-(d+z) \eta_{2}}+4 k^{2}\left(k^{2}+\eta_{2}^{2}\right)\left(k^{2}+A_{1} \frac{p^{2}}{c_{\mathrm{s}}^{2}}\right) \mathrm{e}^{-\mathrm{d} \eta_{2}-(d+z) \eta_{1}}
$$$$
L_{5}=k^{2} D\left(\mathrm{e}^{z \eta_{1}}-\mathrm{e}^{z \eta_{2}}\right)
$$$$
L_{6}=-\left[g\left(k^{2}+A_{1} \frac{p^{2}}{c_{s}^{2}}\right)+A_{1} \frac{p^{2}}{c_{s}^{2}} D\right] \mathrm{e}^{-(2 d+z) \eta_{2}}-k^{2} g \mathrm{e}^{-(2 d+z) \eta_{1}}
$$$$
L_{7}=k^{2} D \mathrm{e}^{z \eta_{1}}-\left(k^{2}+2 A_{1} \frac{p^{2}}{c_{s}^{2}}\right) D \mathrm{e}^{z \eta_{2}}
$$$$
L_{8}=\left[A_{2} \frac{p^{2}}{c_{s}^{2}} D-\left(k^{2}+A_{2} \frac{p^{2}}{c_{s}^{2}}\right) g\right] \mathrm{e}^{-(2 d+z) \eta_{1}}-k^{2} g \mathrm{e}^{-(2 d+z) \eta_{1}}
$$$$
L_{9}=4 k^{2} \eta_{1} \eta_{2}\left(k^{2}+\eta_{2}^{2}\right) \mathrm{e}^{-\mathrm{d} \eta_{1}-(d+z) \eta_{2}}+4 k^{2}\left(k^{2}+\eta_{2}^{2}\right)\left(k^{2}+A_{2} \frac{p^{2}}{c_{s}^{2}}\right) \mathrm{e}^{-\mathrm{d} \eta_{2}-(d+z) \eta_{1}}
$$

$$
L_{10}=-\left[\left(k^{2}+A_{2} \frac{p^{2}}{c_{s}^{2}}\right) g+A_{2} \frac{p^{2}}{c_{s}^{2}} D\right] \mathrm{e}^{-(2 d+z) \eta_{2}}-k^{2} g \mathrm{e}^{-(2 d+z) \eta_{1}}
$$$$
L_{11}=D\left[k^{2} \mathrm{e}^{z \eta_{1}}-\left(k^{2}+2 A_{2} \frac{p^{2}}{c_{s}^{2}}\right) \mathrm{e}^{z \eta_{2}}\right]
$$$$
L_{12}=\left[\eta_{1} \eta_{2} g+\frac{p^{2}}{c_{s}^{2}} \frac{\eta_{1}}{\eta_{2}} D\right] \mathrm{e}^{-(2 d+z) \eta_{2}}+k^{2} g \mathrm{e}^{-(2 d+z) \eta_{1}}
$$$$
L_{13}=-4 k^{2} \eta_{1} \eta_{2}\left(k^{2}+\eta_{2}^{2}\right) \mathrm{e}^{-\mathrm{d} \eta_{1}-(d+z) \eta_{2}}-4 k^{2} \eta_{1} \eta_{2}\left(k^{2}+\eta_{2}^{2}\right) \mathrm{e}^{-\mathrm{d} \eta_{2}-(d+z) \eta_{1}}
$$ 


$$
\begin{aligned}
L_{14} & =D\left[\left(\eta_{1} \eta_{2}+\frac{p^{2}}{c_{s}^{2}} \frac{\eta_{1}}{\eta_{2}}\right) \mathrm{e}^{z \eta_{2}}-k^{2} \mathrm{e}^{z \eta_{1}}\right] \\
L_{15} & =\left[\eta_{1} \eta_{2} g-\frac{p^{2}}{c_{s}^{2}} \frac{\eta_{1}}{\eta_{2}} D\right] \mathrm{e}^{-(2 d+z) \eta_{2}}+k^{2} g \mathrm{e}^{-(2 d+z) \eta_{1}} \\
L_{16} & =D\left[\left(\eta_{1} \eta_{2}-\frac{p^{2}}{c_{s}^{2}} \frac{\eta_{1}}{\eta_{2}}\right) \mathrm{e}^{z \eta_{2}}-k^{2} \mathrm{e}^{z \eta_{1}}\right] \\
g & =\left(k^{2}+\eta_{2}^{2}\right)^{2}+4 k^{2} \eta_{1} \eta_{2}, \quad D=\left(k^{2}+\eta_{2}^{2}\right)^{2}-4 k^{2} \eta_{1} \eta_{2} \\
A_{1} & =\frac{f_{1} n_{3}}{f_{1} n_{3}+f_{3} n_{1}}, \quad A_{2}=\frac{f_{2} n_{3}}{f_{2} n_{3}+f_{3} n_{2}} .
\end{aligned}
$$

Attention is now confined to the surface displacements and to a ramp time-dependence, i.e.

$$
h(t)= \begin{cases}0, & t<q \\ \frac{t}{q}, & 0<t<q . \\ 1, & t>q\end{cases}
$$

Moreover, only solids for which $\lambda=\mu$ are treated. $\uparrow$ Then (16)-(18) yield, on making the variable change $c_{s} k=p x$,

$$
\begin{aligned}
& 4 \pi \rho c_{s}^{3} Q R \bar{u}_{r}^{0}=-n_{3} f_{3}\left(\bar{I}_{1}-\bar{I}_{2}\right) \\
& 4 \pi \rho c_{s}^{3} Q R \bar{u}_{r}^{1}=A_{3}\left[\bar{I}_{3}-\bar{I}_{4}+\bar{I}_{5}-\bar{I}_{6}-2\left(A_{1}+A_{2}\right)\left(\bar{I}_{3}-\bar{I}_{4}-\bar{I}_{7}\right)\right] \\
& 4 \pi \rho c_{s}^{3} Q R \bar{u}_{r}^{2}=G_{1}\left(\bar{I}_{8}-\bar{I}_{9}+\bar{I}_{10}-\bar{I}_{11}\right) \\
& \tilde{I}_{1}=\int_{0}^{\infty} \frac{2 x^{2}\left(3 x^{2}+2 / 3\right) \alpha_{2}}{m_{1}} J_{1}(\delta) \exp \left(-p \mathrm{~d} \alpha_{1} / c_{s}\right) \mathrm{d} x \\
& \bar{I}_{2}=\int_{0}^{\infty} \frac{3 x^{2}\left(2 x^{2}+1\right) \alpha_{2}}{m_{1}} J_{1}(\delta) \exp \left(-p \mathrm{~d} \alpha_{2} / c_{s}\right) \mathrm{d} x \\
& \bar{I}_{3}=\int_{0}^{\infty} \frac{2 x^{3} \alpha_{1} \alpha_{2}}{m_{1}} J_{2}(\delta) \exp \left(-p \mathrm{~d} \alpha_{2} / c_{s}\right) \mathrm{d} x \\
& \bar{I}_{4}=\int_{0}^{\infty} \frac{x^{3}\left(1+2 x^{2}\right)}{m_{1}} J_{2}(\delta) \exp \left(-p \mathrm{~d} \alpha_{2} / c_{s}\right) \mathrm{d} x \\
& \bar{I}_{5}=\int_{0}^{\infty} \frac{x^{3}\left(1+2 x^{2}\right)}{m_{1}} J_{0}(\delta) \exp \left(-p \mathrm{~d} \alpha_{2} / c_{s}\right) \mathrm{d} x \\
& \bar{I}_{6}=\int_{0}^{\infty} \frac{2 x^{3} \alpha_{1} \alpha_{2}}{m_{1}} J_{0}(\delta) \operatorname{cxp}\left(-p \mathrm{~d} \alpha_{1} / c_{s}\right) \mathrm{d} x \\
& \bar{I}_{7}=\int_{0}^{\infty} \frac{x\left(1+2 x^{2}+m_{1}\right)}{m_{1}} J_{0}(\delta) \exp \left(-p \mathrm{~d} \alpha_{2} / c_{s}\right) \mathrm{d} x
\end{aligned}
$$

$\dagger$ Wit condition, the roots of the Rayleigh wave equation are real. This results in simpler algebra later. 


$$
\begin{aligned}
\vec{I}_{8}= & \int_{0}^{\infty} \frac{x^{2}}{\alpha_{2}} J_{1}(\delta) \exp \left(-p \mathrm{~d} \alpha_{2} / c_{s}\right) \mathrm{d} x+3 \bar{I}_{2} \\
\bar{I}_{9}= & \int_{0}^{\infty} \frac{2 x^{4} \alpha_{2}}{m_{1}} J_{1}(\delta) \exp \left(-p \mathrm{~d} \alpha_{2} / c_{s}\right) \mathrm{d} x \\
\bar{I}_{10}= & \int_{0}^{\infty} \frac{x^{4}\left(1+2 x^{2}-4 \alpha_{1} \alpha_{2}\right)}{m_{1} \alpha_{2}} J_{3}(\delta) \exp \left(-p \mathrm{~d} \alpha_{2} / c_{s}\right) \mathrm{d} x \\
\bar{I}_{11}= & \int_{0}^{\infty} \frac{2 x^{4} \alpha_{2}}{m_{1}} J_{3}(\delta) \exp \left(-p \mathrm{~d} \alpha_{1} / c_{s}\right) \mathrm{d} x \\
A_{3}= & 4\left[\left(f_{3} \eta_{1}+f_{1} \eta_{3}\right) \cos \phi+\left(f_{2} \eta_{3}+f_{3} \eta_{2}\right) \sin \phi\right] \\
\alpha_{1}-\sqrt{ }\left(x^{3}+\frac{1}{3}\right), & \alpha_{2}-\sqrt{ }\left(x^{2}+1\right), \quad \delta-p x r / c_{s}, r Q-q c_{s} \\
& m_{1}=\left(1+2 x^{2}\right)^{2}-4 x^{2}\left(x^{2}+1\right)^{\frac{1}{2}}\left(x^{2}+\frac{1}{3}\right)^{\frac{1}{2}} .
\end{aligned}
$$

A typical integral in $(20)-(22)$ is

$$
\bar{S}_{n}=\frac{1}{2} \int_{0}^{\infty} x^{n+1}\left[H_{n}^{(1)}(\delta)+H_{n}^{(2)}(\delta)\right] F(x) \mathrm{d} x
$$

where

$$
F(x)=F_{1}(x) \exp \left(-p \mathrm{~d} \alpha_{1} / c_{s}\right)
$$

$F_{1}(x)$ being free of exponentials and the Bessel functions having been replaced by their Hankel function equivalents. In general the only singularities of $F_{1}(x)$ are branch points at $x= \pm i / \sqrt{ } 3, \pm i$ and simple poles at $\pm \gamma, \gamma=\frac{1}{2}(3+\sqrt{ } 3)^{\frac{1}{2}}$. Integrals of the type (23) have been treated by Pekeris [5] and Aggarwal and Ablow [15] using contour integration techniques and their method will be followed here. When $H_{n}^{(1)}$ arises, the contour consists of the positive real and imaginary axes and a large quarter circle in the first quadrant. For $H_{n}^{(2)}$, a contour consisting of the positive real axis, the negative imaginary axis and a large quarter circle in the fourth quadrant is taken. Aggarwal and Ablow [15] showed that the integrals over the quarter circles go to zero if

$$
F(x)=O\left(|x|^{-\left(n+\frac{1}{2}\right)}\right)
$$

for $|x|$ large and $\arg x \leq \pi / 2$. It is important to note that this order condition is not satisfied by several of the $F_{1}$ 's that arise in the present work. However, thanks to the exponential factor, the corresponding $F$ 's do satisfy the condition, since $\operatorname{Re} \alpha_{1}>0$, when $\operatorname{Re} p>0$ - a restriction that is permissible in view of Lerch's theorem. It is interesting that in order to obtain results for a surface dipole, $d$ cannot be set equal to zero at this stage.

The contour integration method applied to (23) gives

$$
\bar{S}_{n}=\frac{2}{\pi} I \int_{0}^{\infty} v^{n+1} F_{1}(i v) \exp \left(p r v / c_{s}\right) K_{n}\left(\frac{p r v}{c_{s}}\right) \exp \left(-p r / c_{s}\right)\left[l\left(\frac{1}{3}-v^{2}\right)^{\frac{1}{2}}+v\left(1-l^{2}\right)^{\frac{1}{2}}\right] \mathrm{d} v
$$

where $l=d / R, K$ denotes a modified Bessel function of the first kind and $I$ stands for imaginary part. Also, the path of integration is to be interpreted as including an indentation over a simple pole (corresponding to the Rayleigh wave). Equation (24) can be reduced further on 
mapping the $v$-plane onto a $w$-plane by means of the transformation

$$
w=l\left(\frac{1}{3}-v^{2}\right)^{\frac{1}{2}}+v\left(1-l^{2}\right)^{\frac{1}{2}}
$$

The new path of integration can be "traded" for one along the real w-axis by means of Cauchy's theorem. One gets

$$
\bar{S}_{n}=-\frac{2}{\pi} I \int_{0}^{\infty} \exp \left(p r v(w) / c_{s}\right) K_{n}\left[\frac{p}{c_{s}} r v(w)\right] \exp \left(-p r w / c_{s}\right) F_{1}[i v(w)] v^{n+1}(w) \frac{\mathrm{d} v}{\mathrm{~d} w} \mathrm{~d} w
$$

The advantage of this form is that now $w$ is real, positive, monotonic increasing and has an infinite range. Thus it can be interpreted as time. Then, using the shift theorem of the Laplace transform and a result in Erdelyi [20], one gets

$$
L^{-1}\left[\exp \left(p r v / c_{s}\right) K_{n}\left(\frac{p r v}{c_{s}}\right) \exp \left(-p r w / c_{s}\right)\right]=\frac{c_{s} \cosh \Theta_{1}}{R(\tau-w) \Theta_{2}} H\left[\frac{R}{c_{s}}(\tau-w)\right]
$$

where

$$
\begin{aligned}
& \Theta_{1}=n \cosh ^{-1}\left[1+\frac{\tau-w}{v(w)\left(1-l^{2}\right)^{\frac{1}{2}}}\right] \\
& \Theta_{2}-\left[\tau+w-2 l^{2} w-2 l \sqrt{ }\left\{\left(1-l^{2}\right)\left(\frac{1}{3}-w^{2}\right)\right\}\right]^{\frac{1}{2}}
\end{aligned}
$$

$L^{-1}$ denotes the inverse Laplace transform, $H$ the Heaviside unit step function and $r \tau=t c_{s}$.

Using (26), (25) gives

$$
S_{n}=-\frac{2 c_{s}}{\pi R} I \int_{l / \sqrt{ } 3}^{\tau} \frac{\left[l w+\sqrt{ }\left\{\left(1-l^{2}\right)\left(\frac{1}{3}-w^{2}\right)\right\}\right] \cosh \Theta_{1} v^{n+1}(w) F_{1}[i v(w)]}{\left[\left(\frac{1}{3}-w^{2}\right)(\tau-w)\right]^{\frac{1}{2}} \Theta_{2}} \mathrm{~d} w .
$$

On considering a surface dipole, i.e. $l=0$, it can be shown that, on employing a partial fraction decomposition, integrals such as (27) can be written in closed form. Applying such results to $(20)-(22)$, one gets

$$
4 \pi \rho c_{s}^{3} Q r u_{r}^{0}= \begin{cases}0, & \tau<1 / \sqrt{ } 3 \\ \beta_{1}(\tau), \quad 1 / \sqrt{ } 3<\tau<Q+1 / \sqrt{ } 3 \\ \beta_{1}(\tau)-\beta_{1}(\tau-Q), & Q+1 / \sqrt{ } 3<\tau<1 \\ \beta_{2}(\tau)-\beta_{1}(\tau-Q), & 1<\tau<\gamma \\ \beta_{3}(\tau)-\beta_{1}(\tau-Q), & \gamma<\tau<1+Q \\ \beta_{3}(\tau)-\beta_{2}(\tau-Q), & 1+Q<\tau<\gamma+Q \\ \beta_{3}(\tau)-\beta_{3}(\tau-Q), & \tau>\gamma+Q\end{cases}
$$




$$
\begin{aligned}
& \frac{4 \pi \rho c_{s}^{3} Q r}{A_{3}} u_{r}^{1}= \begin{cases}0, & \tau<1 / \sqrt{ } 3 \\
\beta_{4}(\tau), \quad 1 / \sqrt{ } 3<\tau<Q+1 / \sqrt{ } 3 \\
\beta_{4}(\tau)-\beta_{4}(\tau-Q), & Q+1 / \sqrt{3}<\tau<1 \\
\beta_{5}(\tau)-\beta_{4}(\tau-Q), & 1<\tau<\gamma \\
\beta_{6}(\tau)-\beta_{4}(\tau-Q), & \gamma<\tau<1+Q \\
\beta_{6}(\tau)-\beta_{5}(\tau-Q), & 1+Q<\tau<\gamma+Q \\
\beta_{6}(\tau)-\beta_{6}(\tau-Q), & \tau>\gamma+Q\end{cases} \\
& \frac{4 \pi \rho c_{s}^{3} Q r}{G_{1}} u_{r}^{2}= \begin{cases}0, & \tau<1 / \sqrt{ } 3, \\
\beta_{7}(\tau), \quad 1 / \sqrt{ } 3<\tau<Q+1 / \sqrt{ } 3 \\
\beta_{7}(\tau)-\beta_{7}(\tau-Q), & Q+1 / \sqrt{ } 3<\tau<1 \\
\beta_{8}(\tau)-\beta_{7}(\tau-Q), & 1<\tau<\gamma \\
\beta_{9}(\tau)-\beta_{7}(\tau-Q), & \gamma<\tau<1+Q \\
\beta_{9}(\tau)-\beta_{8}(\tau-Q), & 1+Q<\tau<\gamma+Q \\
\beta_{9}(\tau)-\beta_{9}(\tau-Q), & \tau>\gamma+Q\end{cases} \\
& \beta_{1}=\frac{5 c_{s} \tau}{48 r}\left\{\frac{2}{\sqrt{3}}+\frac{3}{\left(\tau^{2}-\frac{1}{4}\right)^{\frac{1}{2}}}-\frac{(1+\sqrt{ } 3)^{\frac{1}{2}}}{\left[\tau^{2}-(3-\sqrt{3}) / 4\right]^{\frac{1}{2}}}-\frac{(-1+\sqrt{3})^{\frac{1}{2}}}{\left[-\tau^{2}+(3+\sqrt{3}) / 4\right]^{\frac{1}{2}}}\right\} \sqrt{3} \\
& \beta_{2}=\frac{5 c_{s} \tau}{24 r}\left\{\frac{2}{\sqrt{3}}-\frac{(-1+\sqrt{3})^{\frac{1}{2}}}{\left[-\tau^{2}+(3+\sqrt{3}) / 4\right]^{\frac{1}{2}}}\right\} \sqrt{3} \\
& \beta_{3}=\frac{5 c_{s} \tau}{12 r} \\
& \beta_{4}=T_{1}+T_{2}-2\left(A_{1}+A_{2}\right)\left(T_{1}+2 T_{2}\right) \\
& \beta_{5}=T_{3}+T_{4}-2\left(A_{1}+A_{2}\right)\left(T_{3}+2 T_{4}\right) \\
& \beta_{6}=\beta_{5}+\frac{c_{s} \tau^{2}}{2 r\left(\tau^{2}-\gamma^{2}\right)^{\frac{1}{2}}}-\frac{\left(A_{1}+A_{2}\right)\left(\tau^{2}+\gamma^{2}\right) c_{s}}{r\left(\tau^{2}-\gamma^{2}\right)^{\frac{1}{2}}} \\
& \beta_{7}=T_{5}+T_{6}, \quad \beta_{8}=T_{7}+T_{8}, \quad \beta_{9}=T_{9}+T_{10}
\end{aligned}
$$

$$
\begin{aligned}
96 \pi r T_{1}= & \left\{\left(24 \tau^{2}-11\right) K(\xi)+8 m(\xi)-9\left(8 \tau^{2}-1\right) \Gamma\left(8 \xi^{2}, \xi\right)\right. \\
& +\left[8(3-2 \sqrt{3}) \tau^{2}+3(-1+\sqrt{3}) \Gamma\left[-(-20+12 \sqrt{3}) \xi^{2}, \xi\right]\right. \\
& \left.+\left[8(3+2 \sqrt{3}) \tau^{2}-3(1+\sqrt{3})\right] \Gamma\left[(20+12 \sqrt{3}) \xi^{2}, \xi\right]\right\} c_{s} \sqrt{ }(3 / 2) \\
16 \pi r T_{2}= & -\left\{-11 K(\xi)+8 m(\xi)+9 \Gamma\left(8 \xi^{2}, \xi\right)-3(1+\sqrt{3}) \Gamma\left[(20+12 \sqrt{3}) \xi^{2}, \xi\right]\right. \\
& \left.+3(-1+\sqrt{3}) \Gamma\left[-(-20+12 \sqrt{3}) \xi^{2}, \xi\right]\right\} c_{s} \sqrt{ }(3 / 2) \\
96 \pi r T_{3}= & \left\{8 m\left(\xi_{1}\right) / \xi_{1}^{2}+\left[24 \tau^{2}-\left(3+8 / \xi_{1}^{2}\right)\right] K\left(\xi_{1}\right)-9\left(8 \tau^{2}-1\right) \Gamma\left(8, \xi_{1}\right)\right. \\
& +\left[8(3-2 \sqrt{3}) \tau^{2}+3(-1+\sqrt{3})\right] \Gamma\left[-(-20+12 \sqrt{3}), \xi_{1}\right] \\
& \left.+\left[8(3+2 \sqrt{3}) \tau^{2}-3(1+\sqrt{3})\right] \Gamma\left[(20+12 \sqrt{3}), \xi_{1}\right]\right\} c_{s} \xi_{1} \sqrt{ }(3 / 2)
\end{aligned}
$$




$$
\begin{aligned}
& 16 \pi r T_{4}=-\left\{8 m\left(\xi_{1}\right) / \xi_{1}^{2}-\left(8+3 \xi_{1}^{2}\right) K\left(\xi_{1}\right) / \xi_{1}^{2}+9 \Gamma\left(8, \xi_{1}\right)-3(1+\sqrt{3}) \Gamma\left[(20+12 \sqrt{ } 3), \xi_{1}\right]\right. \\
& \left.+3(-1+\sqrt{ } 3) \Gamma\left[-(-20+12 \sqrt{ } 3), \xi_{1}\right]\right\} c_{s} \xi_{1} \sqrt{ }(3 / 2) \\
& 16 r T_{5}=\left\{\frac{2}{\sqrt{3}}+\frac{3}{\left(\tau^{2}-\frac{1}{4}\right)^{\frac{1}{2}}}-\frac{(1+\sqrt{3})^{\frac{1}{2}}}{\left[\tau^{2}-(3-\sqrt{3}) / 4\right]^{\frac{1}{2}}}-\frac{(-1+\sqrt{ } 3)^{\frac{1}{2}}}{\left[-\tau^{2}+(3+\sqrt{3}) / 4\right]^{\frac{1}{2}}}\right\} c_{s} \tau \sqrt{ } 3 \\
& \pi r T_{6}=-2 c_{s} \tau\left(48 \tau^{2} \Psi_{1}-3 \Psi_{2}\right) \\
& 8 r T_{7}=\left\{\frac{10}{\sqrt{3}}-\frac{(-1+\sqrt{ } 3)^{\frac{1}{2}}}{\left[-\tau^{2}+(3+\sqrt{ } 3) / 4\right]^{\frac{1}{2}}}\right\} c_{s} \tau \sqrt{ } 3 \\
& \pi T_{8}=-2 c_{s} \tau\left(-3 \Psi_{2}+48 \tau^{2} \Psi_{1}+4 \tau^{2} \Psi_{3}-3 \Psi_{4}\right), 4 r T_{9}=5 c_{s} \tau \\
& -\pi r T_{10}=-2 c_{s} \tau\left(-3 \Psi_{5}+48 \tau^{2} \Psi_{6}+4 \tau^{2} \Psi_{7}-3 \Psi_{8}\right) \\
& 32 \Psi_{1 \sqrt{ } 2}=3 \pi\left\{\frac{3 \sqrt{ } 2}{\left(\tau^{2}-\frac{1}{4}\right)^{\frac{1}{2}}}-\frac{[5+3 \sqrt{ } 3]^{\frac{1}{2}}}{\left[\tau^{2}-(3-\sqrt{3}) / 4\right]^{\frac{1}{2}}}+\frac{[-5+3 \sqrt{3}]^{\frac{1}{2}}}{\left[-\tau^{2}+(3+\sqrt{3}) / 4\right]^{\frac{1}{2}}}\right\} \\
& 32 \Psi_{2}=\left\{\frac{1}{2 \sqrt{3}}+\frac{3}{\left(\tau^{2}-\frac{1}{4}\right)^{\frac{1}{2}}}-\frac{(1+\sqrt{3})^{\frac{1}{2}}}{\left[\tau^{2}-(3-\sqrt{3}) / 4\right]^{\frac{1}{2}}}+\frac{(-1+\sqrt{3})^{\frac{1}{2}}}{\left[-\tau^{2}+(3+\sqrt{3}) / 4\right]^{\frac{1}{2}}}\right\} \pi \sqrt{3} \\
& 8 \Psi_{3}=\pi\left\{-\frac{3 \sqrt{ } 3}{\left(\tau^{2}-\frac{1}{4}\right)^{\frac{1}{2}}}+\frac{2(1+\sqrt{ } 3)^{\frac{1}{2}}}{\left[\tau^{2}-(3-\sqrt{3}) / 4\right]^{\frac{1}{2}}}+\frac{2(-1+\sqrt{ } 3)^{\frac{1}{2}}}{\left[-\tau^{2}+(3+\sqrt{3}) / 4\right]^{\frac{1}{2}}}\right\} \\
& 32 \Psi_{4}=-\left\{\frac{10}{\sqrt{ } 3}+\frac{3}{\left(\tau^{2}-\frac{1}{4}\right)^{\frac{1}{2}}}-\frac{(1+\sqrt{3})^{\frac{1}{2}}}{\left[\tau^{2}-(3-\sqrt{3}) / 4\right]^{\frac{1}{2}}}+\begin{array}{c}
(-1+\sqrt{ } 3)^{\frac{1}{2}} \\
{\left[-\tau^{2}+(3+\sqrt{3}) / 4\right]^{\frac{1}{2}}}
\end{array}\right\} \pi \sqrt{3} \\
& 32 \Psi_{5}=\left\{\frac{1}{2 \sqrt{3}}+\frac{3}{\left(\tau^{2}-\frac{1}{4}\right)^{\frac{1}{2}}}-\frac{(1+\sqrt{3})^{\frac{1}{2}}}{\left[\tau^{2}-(3-\sqrt{3}) / 4\right]^{\frac{1}{2}}}\right\} \pi \sqrt{ } 3 \\
& 32 \Psi_{6 \sqrt{ }} 2=3 \pi\left\{\frac{3 \sqrt{ } 2}{\left(\tau^{2}-\frac{1}{4}\right)^{\frac{1}{2}}}-\frac{(5+3 \sqrt{ } 3)^{\frac{1}{2}}}{\left[\tau^{2}-(3-\sqrt{3}) / 4\right]^{\frac{1}{2}}}\right\} \\
& 8 \Psi_{7}=\pi\left\{-\frac{3 \sqrt{ } 3}{\left(\tau^{2}-\frac{1}{4}\right)^{\frac{1}{2}}}+\frac{2(1+\sqrt{3})^{\frac{1}{2}}}{\left[\tau^{2}-(3-\sqrt{ } 3) / 4\right]^{\frac{1}{2}}}\right\} \\
& 32 \Psi_{8}=-\left\{\frac{10}{\sqrt{3}}+\frac{3}{\left(\tau^{2}-\frac{1}{4}\right)^{\frac{1}{2}}}-\frac{(1+\sqrt{3})^{\frac{1}{2}}}{\left[\tau^{2}-(3-\sqrt{3}) / 4\right]^{\frac{1}{2}}}\right\} \pi \sqrt{ } 3 \\
& 2 \xi^{2}=\left(3 \tau^{2}-1\right), \quad \xi_{1}=1 / \xi
\end{aligned}
$$

and

$$
\begin{aligned}
K(\xi) & =\int_{0}^{\pi / 2} \frac{\mathrm{d} \theta}{\left(1-\xi^{2} \sin ^{2} \theta\right)^{\frac{1}{2}}}, \quad m(\xi)=\int_{0}^{\pi / 2}\left(1-\xi^{2} \sin ^{2} \theta\right)^{\frac{1}{2}} \mathrm{~d} \theta \\
\Gamma(n, \xi) & =\int_{0}^{\pi / 2} \frac{\mathrm{d} \theta}{\left(1+n \sin ^{2} \theta\right)\left(1-\xi^{2} \sin ^{2} \theta\right)^{\frac{1}{2}}}
\end{aligned}
$$

are complete elliptic integral of the first, second and third kinds, respectively. 
Similarly it can be shown that the other displacements are given by

$$
\begin{aligned}
& 4 \pi \rho c_{s}^{3} Q r u_{\phi}^{0}=\left(n_{1} f_{2}-n_{2} f_{1}\right)\left\{\begin{array}{l}
0, \quad \tau<1 \\
\beta_{10}(\tau), \quad 1<\tau<1+Q \\
\beta_{10}(\tau)-\beta_{10}(\tau-Q), \quad \tau>1+Q
\end{array}\right. \\
& \frac{4 \pi \rho c_{s}^{3} Q r}{A_{4}} u_{\phi}^{1}= \begin{cases}0, \quad \tau<1 / \sqrt{ } 3 \\
\beta_{11}(\tau), \quad 1 / \sqrt{ } 3<\tau<Q+1 / \sqrt{ } 3 \\
\beta_{11}(\tau)-\beta_{11}(\tau-Q), & Q+1 / \sqrt{ } 3<\tau<1 \\
\beta_{12}(\tau)-\beta_{11}(\tau-Q), & 1<\tau<\gamma \\
\beta_{13}(\tau)-\beta_{11}(\tau-Q), & \gamma<\tau<1+Q \\
\beta_{13}(\tau)-\beta_{12}(\tau-Q), & 1+Q<\tau<\gamma+Q \\
\beta_{13}(\tau)-\beta_{13}(\tau-Q), & \tau>\gamma+Q\end{cases} \\
& \frac{4 \pi \rho c_{s}^{3} Q r}{G_{2}} u_{\phi}^{2}= \begin{cases}0, \quad \tau<1 / \sqrt{ } 3 \\
\beta_{14}(\tau) \quad 1 / \sqrt{3}<\tau<Q+1 / \sqrt{ } 3 \\
\beta_{14}(\tau)-\beta_{14}(\tau-Q), & Q+1 / \sqrt{ } 3<\tau<1 \\
\beta_{15}(\tau)-\beta_{14}(\tau-Q), & 1<\tau<\gamma \\
\beta_{16}(\tau)-\beta_{14}(\tau-Q), & \gamma<\tau<1+Q \\
\beta_{14}(\tau)-\beta_{15}(\tau-Q), & 1+Q<\tau<\gamma+Q \\
\beta_{16}(\tau)-\beta_{16}(\tau-Q), & \tau>\gamma+Q\end{cases} \\
& \frac{4 \pi \rho c_{s}^{3} Q r}{f_{3} n_{3}} u_{z}^{0}= \begin{cases}0, \quad \tau<1 / \sqrt{ } 3 \\
\beta_{17}(\tau), \quad 1 / \sqrt{ } 3<\tau<Q+1 / \sqrt{ } 3 \\
\beta_{17}(\tau)-\beta_{17}(\tau-Q), & Q+1 / \sqrt{ } 3<\tau<1 \\
\beta_{18}(\tau)-\beta_{17}(\tau-Q), & 1<\tau<\gamma \\
\beta_{19}(\tau)-\beta_{17}(\tau-Q), & \gamma<\tau<1+Q \\
\beta_{19}(\tau)-\beta_{18}(\tau-Q), & 1+Q<\tau<\gamma+Q \\
\beta_{19}(\tau)-\beta_{19}(\tau-Q), & \tau>\gamma+Q\end{cases} \\
& \frac{4 \pi \rho c_{s}^{3} Q r u_{z}^{1}}{A_{5}}= \begin{cases}\beta_{20}(\tau), \quad 1 / \sqrt{ } 3<\tau<Q+1 / \sqrt{ } 3 \\
\beta_{20}(\tau)-\beta_{20}(\tau-Q), & Q+1 / \sqrt{ } 3<\tau<1 \\
\beta_{21}(\tau)-\beta_{20}(\tau-Q), & 1<\tau<\gamma \\
\beta_{22}(\tau)-\beta_{20}(\tau-Q), & \gamma<\tau<1+Q \\
\beta_{22}(\tau)-\beta_{21}(\tau-Q), & 1+Q<\tau<\gamma+Q \\
\beta_{22}(\tau)-\beta_{22}(\tau-Q), & \tau>\gamma+Q\end{cases}
\end{aligned}
$$




$$
\begin{aligned}
& \frac{8 \pi \rho c_{s}^{3} Q r}{G_{1}} u_{z}^{2}=- \begin{cases}0, \quad \tau<1 / \sqrt{3} \\
\beta_{23}(\tau), \quad 1 / \sqrt{3}<\tau<Q+1 / \sqrt{ } 3 \\
\beta_{23}(\tau)-\beta_{23}(\tau-Q), & Q+1 / \sqrt{3}<\tau<1 \\
\beta_{24}(\tau)-\beta_{23}(\tau-Q), & 1<\tau<\gamma \\
\beta_{25}(\tau)-\beta_{23}(\tau-Q), & \gamma<\tau<1+Q \\
\beta_{25}(\tau)-\beta_{24}(\tau-Q), & 1+Q<\tau<\gamma+Q \\
\beta_{25}(\tau)-\beta_{25}(\tau-Q), & \tau>\gamma+Q\end{cases} \\
& A_{4}=-2\left[\left(f_{2} n_{3}+f_{3} n_{2}\right) \cos \phi-\left(f_{1} n_{3}+f_{3} n_{1}\right) \sin \phi\right] \\
& A_{5}=2\left[\left(f_{1} n_{3}-f_{3} n_{1}\right) \cos \phi+\left(f_{2} n_{3}-f_{3} n_{2}\right) \sin \phi\right] \\
& r \beta_{10}=c_{s} \tau, \quad \beta_{11}=T_{1}-T_{2}-2\left(A_{1}+A_{2}\right)\left(T_{1}-2 T_{2}\right) \\
& \beta_{12}=T_{3}-T_{4}-2\left(A_{1}+A_{2}\right)\left(T_{3}-2 T_{4}\right) \\
& \beta_{13}=\beta_{12}+\frac{c_{s}}{2 r}\left(\tau^{2}-r^{2}\right)^{\frac{1}{2}}-\frac{\left(A_{1}+A_{2}\right) c_{s}}{r}\left(\tau^{2}-\gamma^{2}\right)^{\frac{1}{2}} \\
& \beta_{14}=T_{5}-T_{6}, \quad \beta_{15}=T_{7}-T_{8}, \quad \beta_{16}=T_{9}-T_{10}
\end{aligned}
$$

$48 \pi r \beta_{1\rceil}-\left\{-8 m(\xi)+11 K(\xi)+3(1+\sqrt{3}) \Gamma\left[(20+12 \sqrt{3}) \xi^{2}, \xi\right]\right.$

$$
\left.-3(-1+\sqrt{3}) \Gamma\left[-(-20+12 \sqrt{3}) \xi^{2}, \xi\right]-9 \Gamma\left(8 \xi^{2}, \xi\right)\right\} 5 c_{s} \sqrt{ }(3 / 2)
$$

$$
\begin{gathered}
48 \pi r \beta_{18}=-\left\{\frac{8 m\left(\xi_{1}\right)}{\xi_{1}^{2}}+\left(3+\frac{8}{\xi_{1}^{2}}\right) K\left(\xi_{1}\right)+3(1+\sqrt{3}) \Gamma\left[(20+12 \sqrt{3}), \xi_{1}\right]\right. \\
\left.-3(-1+\sqrt{3}) \Gamma\left[-(-20+12 \sqrt{3}), \xi_{1}\right]-9 \Gamma\left(8, \xi_{1}\right)\right\} 5 c_{s} \xi_{1} \sqrt{(3 / 2)} \\
\beta_{19}=\beta_{18}-\frac{5 \gamma^{2} c_{s}}{r\left(\tau^{2}-y^{2}\right)^{\frac{1}{2}}}
\end{gathered}
$$

$$
\begin{aligned}
8 r \beta_{20}= & -c_{s} \tau\left\{6-\frac{\sqrt{3}}{\left(\tau^{2}-\frac{1}{4}\right)^{\frac{1}{2}}}-\frac{(5+3 \sqrt{3})^{\frac{1}{2}}}{\left[-\tau^{2}+(3+\sqrt{3}) / 4\right]^{\frac{1}{2}}}+\frac{(-5+3 \sqrt{3})^{\frac{1}{2}}}{\left[\tau^{2}-(3-\sqrt{3}) / 4\right]^{\frac{1}{2}}}\right\} \\
4 r \beta_{21}= & -c_{s} \tau\left\{6-\frac{(5+3 \sqrt{3})^{\frac{1}{2}}}{\left[-\tau^{2}+(3+\sqrt{3}) / 4\right]^{\frac{1}{2}}}\right\}, \quad 4 r \beta_{22}=-c_{s} \tau \\
\pi r \beta_{23}= & 12 c_{s}\left(2 \tau^{2} T_{11}-T_{12}\right), \quad \pi r \beta_{24}=12 c_{s}\left(2 \tau^{2} T_{13}-T_{14}\right) \\
48 T_{11}= & \left\{3 K(\xi)-9 \Gamma\left(8 \xi^{2}, \xi\right)+(3-2 \sqrt{3}) \Gamma\left[-(-20+12 \sqrt{3}) \xi^{2}, \xi\right]\right. \\
& \left.+(3+2 \sqrt{ } 3) \Gamma\left[(20+12 \sqrt{ } 3) \xi^{2}, \xi\right]\right\} \sqrt{ }(3 / 2) \\
192 T_{12}= & -\left\{8 m(\xi)-11 K(\xi)+9 \Gamma\left(8 \xi^{2}, \xi\right)+3(-1+\sqrt{ } 3) \Gamma\left[-(-20+12 \sqrt{ } 3) \xi^{2}, \xi\right]\right. \\
& -3\left(1+\sqrt{ }(3) \Gamma\left[(20+12 \sqrt{ } 3) \xi^{2}, \xi\right]\right\} \sqrt{ }(3 / 2)
\end{aligned}
$$




$$
\begin{aligned}
48 T_{13}= & \left\{3 K\left(\xi_{1}\right)-9 \Gamma\left(8, \xi_{1}\right)+(3-2 \sqrt{3}) \Gamma\left[-(-20+12 \sqrt{3}), \xi_{1}\right]\right. \\
& \left.+(3+2 \sqrt{3}) \Gamma\left[(20+12 \sqrt{ } 3), \xi_{1}\right]\right\} \xi_{1} \sqrt{ }(3 / 2) \\
192 T_{14}= & -\left\{\frac{8 m\left(\xi_{1}\right)}{\xi_{1}^{2}}-\left(3+\frac{8}{\xi_{1}^{2}}\right) K\left(\xi_{1}\right)+9 \Gamma\left(8, \xi_{1}\right)+3(-1+\sqrt{3}) \Gamma\left[-(-20+12 \sqrt{3}), \xi_{1}\right]\right. \\
& \left.-3(1+\sqrt{ } 3) \Gamma\left[(20+12 \sqrt{ } 3), \xi_{1}\right]\right\} \xi_{1} \sqrt{ }(3 / 2) .
\end{aligned}
$$

The above sequence is for $Q=0 \cdot 1$. It could be quite different for different values of $Q$. A main feature of these results (and a major contribution of the paper) is the fact that closedform expressions are obtained for an arbitrary dipole orientation. It is seen that the general response is nonaxisymmetric, with $\cos \phi, \sin \phi, \cos 2 \phi$ and $\sin 2 \phi$ arising.

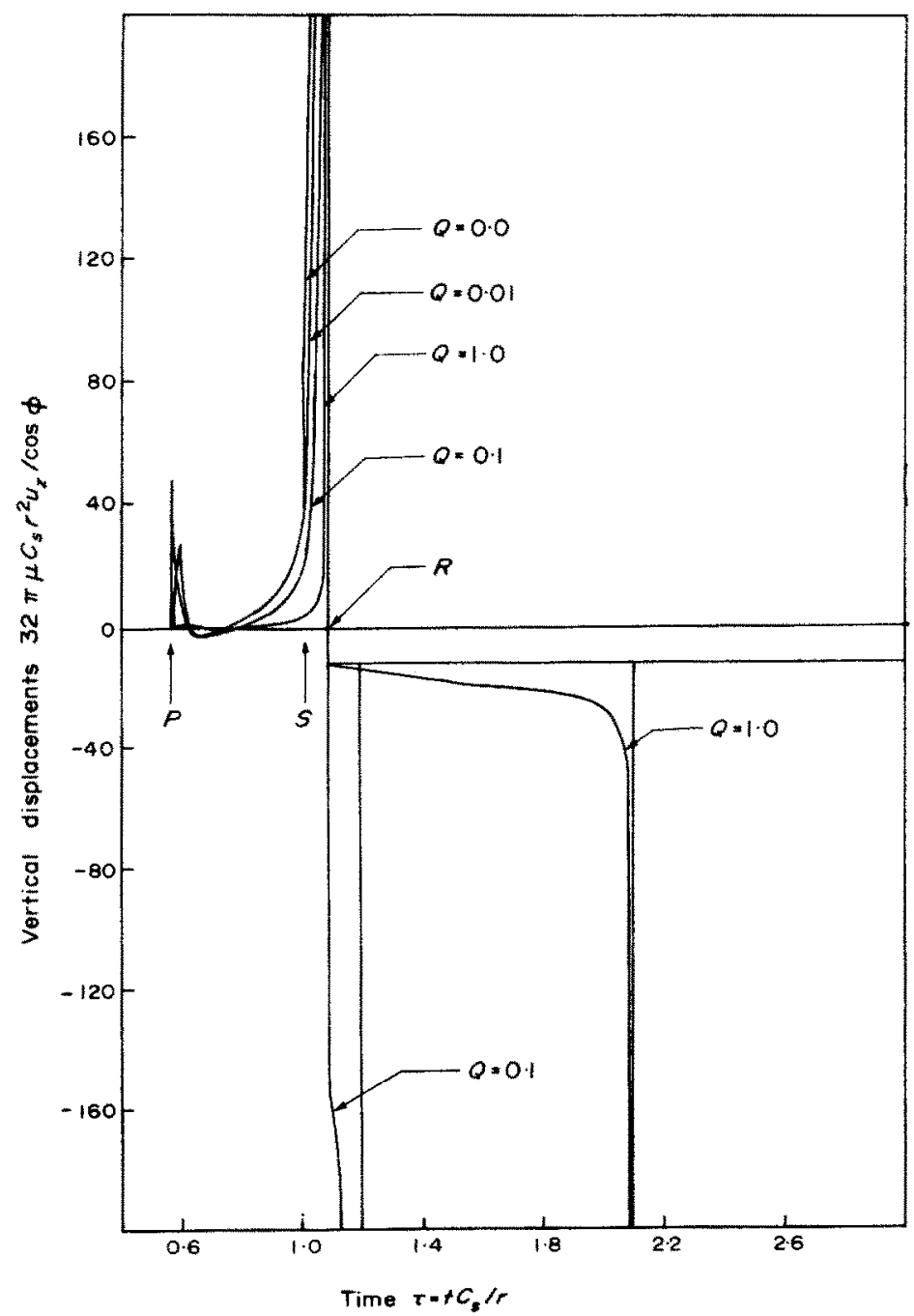

FIG. 2. Vertical displacements $32 \pi \mu c_{s} r^{2} u_{z} / \cos \phi$ vs. time $\tau=t c_{s} / r$ for $Q=0.0,0.01,0.1,1.0$. 


\section{NUMERICAL RESULTS}

Here numerical results based on $(28)-(36)$ are presented and discussed. Only the special geometry,

$$
\begin{array}{lll}
f_{1}=0, & f_{2}=0, & f_{3}=1 \\
n_{1}=1, & n_{2}=0, & n_{3}=0
\end{array}
$$

which corresponds to a vertical dipole with its normal along the $x$-axis, is treated.

Shown in Fig. 2 are the vertical displacements $u_{z}$ as functions of time for several values of the ramp rise-time $Q$. The arrows indicate the arrival of various events. $P$ designates the pressure wave, $S$ the shear wave and $R$ the Rayleigh event. A major feature is the singular behaviour (like $1 / \sqrt{ } \tau$ as $\tau$ goes to 0 ) at the Rayleigh and delayed Rayleigh arrivals. This is in contrast to the case of a point surface load, which was recently treated by the authors [21]. There the vertical displacements were found to be finite for a ramp time-dependence. The growth of a local maximum near the $P$-arrival as $Q$ decreases is also noteworthy. In fact for $Q=0$, which corresponds to a Heaviside step time-dependence, the vertical displacements undergo a finite jump at $P$. A finite jump at $S$ also occurs for $Q=0$. Finally it should be noted that for all $Q$ values, the displacements are constant after the delayed Rayleigh arrival.

Figure 3 gives the horizontal displacements $u_{r}$ as functions of time for several values of $Q$. Again singular behaviour like $1 / \sqrt{ } \tau$ occurs at the Rayleigh and delayed Rayleigh

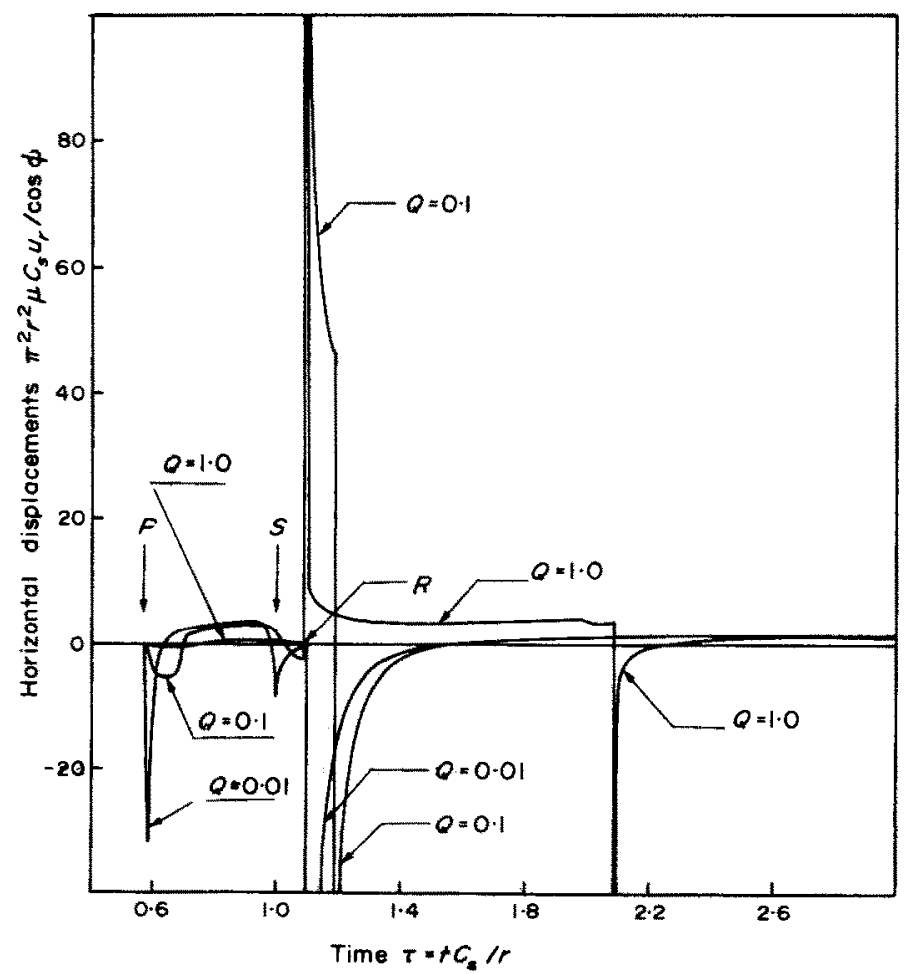

FIG. 3. Horizontal displacements $\pi^{2} r^{2} \mu c_{s} u_{r} / \cos \phi$ vs. time $t=t c_{s} / r$ for $Q=0.01,0-1,1 \cdot 0$. 
arrivals. Also a build up of a local maximum just after the $P$-arrival can be seen as $Q$ tends to zero. Here the displacements are not constant after the delayed Rayleigh arrival, but instead slowly approach their static values.

Figure 4 gives the horizontal displacements $u_{\phi}$ as functions of time for several values of $Q$. In strong contrast to $u_{z}$ and $u_{r}, u_{\phi}$ is not singular for any value of $Q$, except $Q=0$. As $Q$ approaches zero though, infinite jumps at the Rayleigh and delayed Rayleigh arrivals begin to build up.

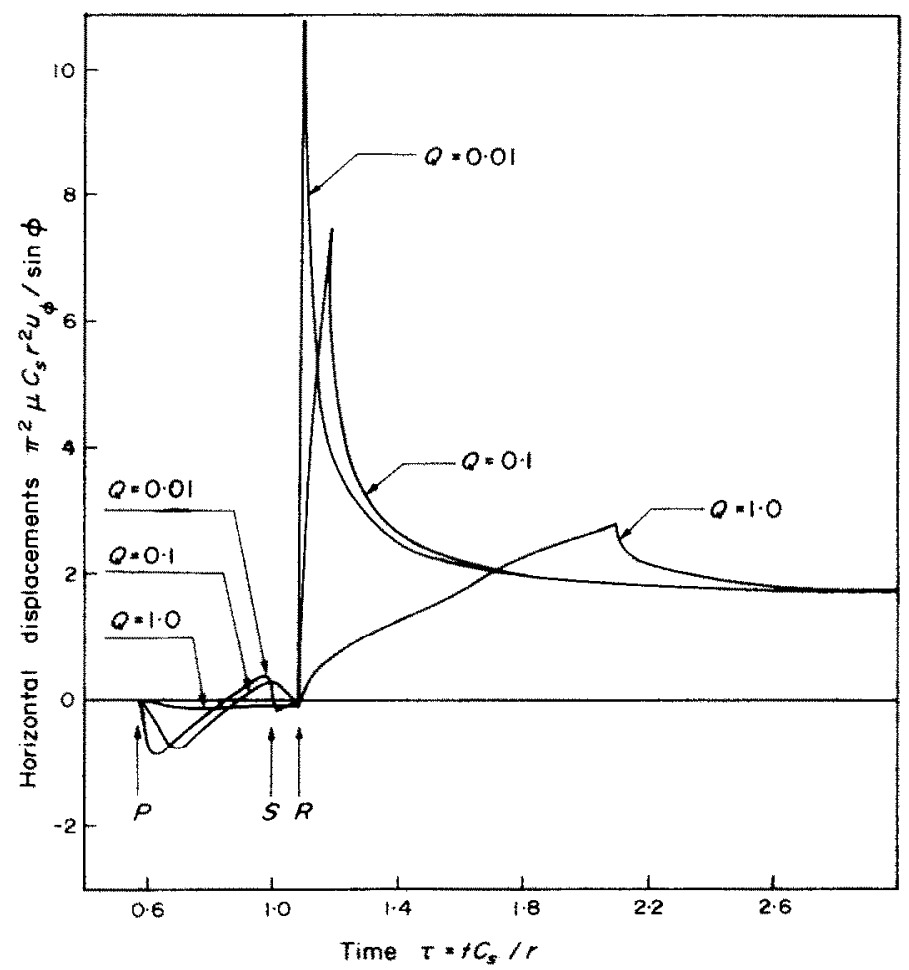

Fro. 4. Horizontal displacements $\pi^{2} \mu c_{s} r^{2} u_{\phi} / \sin \phi$ vs. time $\tau=t c_{s} / r$ for $Q=0.01,0.1$ and 1.0 .

\section{REFERENCES}

[1] R. A. ScotT and J. MikLowITZ, Transient non-axisymmetric wave propagation in an infinite isotropic elastic plate. Int. J. Solids Struct. 5, 65 (1969).

[2] L. KNOPOFF and F, GLBERT, Radiation from strike-slip fault. Bull seism. Soc. Am. 49, 163 (1959).

[3] F. GILRERT and L. KNOPOFF, The directivity problem for a buried line source. J. Geophys. Res. 26, 626 (1961).

[4] R. BURRIDGe, E. R. LAPWOOD and L. KNOPOFF, First motion from seismic sources near a free surface. Bull. seism. Soc. Am. 54, 1889 (1964).

[5] C. L. Pekeris, The seismic surface pulse. Proc. natn. Acad. Sci. 41, 469 (1955).

[6] C. L. Pexeris, Solutions of an integral equation occurring in impulsive wave propagation problems. Proc. natn. Acad. Sci. 42, 439 (1956).

[7] C. C. ChaO, H. H. BLeich and J. SACKMan, Surface waves in an elastic half-space. J. appl. Mech. 28, 300 (1961). 
[8] H. A. LANG, Surface displacements in an elastic half space, Z. angew Math. Mech. 41, 141 (1961).

[9] C. L. Pekeris, The seismic buried pulse. Proc. natn. Acad. Sci. 41, 469, 629 (1955).

[10] C. L. Pekeris and H. Lifson, Motion of the surface of a uniform elastic half-space produced by a buried pulse. J. acoust. Soc. Am. 29, 1233 (1957).

[11] E. PINNEy, Surface motion due to a point source in a semi-infinite elastic medium. Bull. seism. Soc. Am. 44, 571 (1954).

[12] C. L. PEKERIS and I. M. Longman, The motion of the surface of a uniform elastic half-space produced by a buried torque-pulse. Geophys. J. R. astr. Soc. 1, 146 (1958).

[13] C. C. ChaO, Dynamical response of an elastic half-space to tangential surface loadings. J.appl. Mech. 27, $559(1960)$.

[14] H. R. AgGarwal and C. M. Ablow, The extension of the Bateman-Pekeris theorem with application to a wave propagation problem. J, math. Phys. 44, 267 (1965).

[15] H. R. AgGaRwal and C. M. ABLow, Solution to a class of three dimensional pulse propagation problems in an elastic half-space. Int. J. Engng Sci. 5, 663 (1967).

[16] G. EASON, The displacements produced in an elastic half-space by a suddenly applied surface force. J. Inst. Math. Applic. 2, 299 (1966).

[17] M. Mrtra, The disturbances produced by axially-symmetric, time-dependent body forces in an elastic half-space, Proc, natn. Inst. Sci, India 29A, 271 (1963).

[18] U. CHANDRA, Exact solution for the displacement components in $P_{1} P_{2} P_{1},\left(S_{1} S_{2} S_{1}\right)_{S W},\left(S_{1} S_{2} S_{1}\right)_{S H}$ head waves due to an impulsive double couple source. Bull. seism. Soc. Am. 59, 317 (1969).

[19] N. A. HASKELL, Radiation pattern of surface waves from point sources in multilayered medium. Bull. seism. Soc. Am. 54, 377 (1964).

[20] A. Erdelyi, W. Magnus, F. Oberhettinger and F. G. Tricomi, Tables of Integral Transforms, in Bateman Manuscript Project, Vol. 2. McGraw-Hill (1957).

[21] O. F. Afandi and R. A. Scott, A note on dynamic surface displacements in an elastic half-space, Acta Mech. to appear.

(Received 22 Seplember 1971 ; revised 25 February 1972)

\begin{abstract}
Абатракт-B упругом полупространстве исследуются поверхностные перемсщения вслсдствие поверхностного, зависяшего от времени диполя. Даются выражения в замкнутом виде для пронзвольно направленного диполя, с зависимостью наклона во времени. Приводится численный аналих этих выражений для специального направления диполя, для некоторых значений возврастания наклона во времени.
\end{abstract}

